Animal Health Research Institute

Assiut Provincial Laboratory.

\title{
SOME STUDIES ON CLOSTRIDIUM COLINUM INFECTION IN CHICKENS AND QUAILS
}

(With One Table and 27 Figures)

\author{
By

\section{HEBAT-ALLAH A.E. MOHAMED and I.A. FOUAD}

(Received at 1/4/2009)

بعض الاراسات عن الإصابة بالكلستيريديوم كولينم في الاجاج والسمان هبة الله عبل الحليم محدد ، إبراهيم أحدد فؤاد

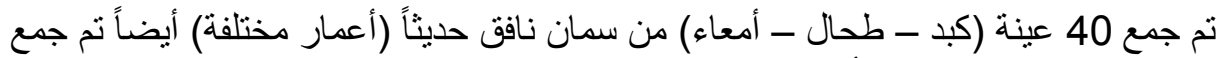

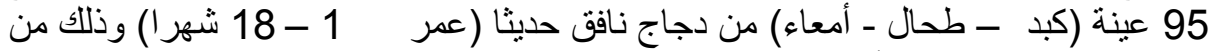

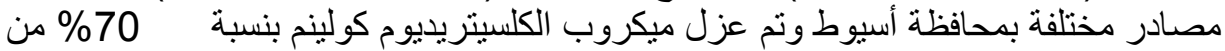

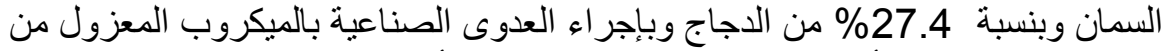

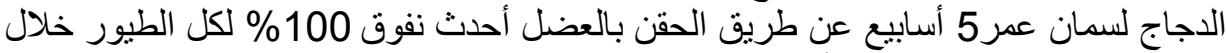

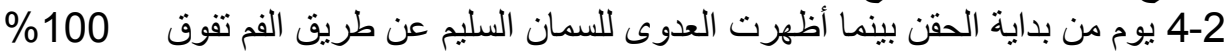

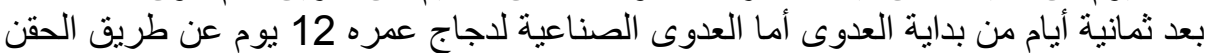

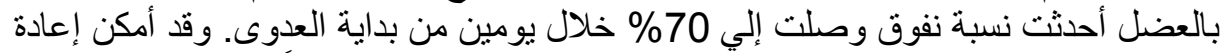

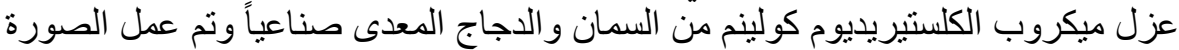

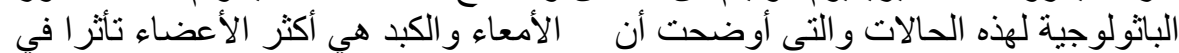

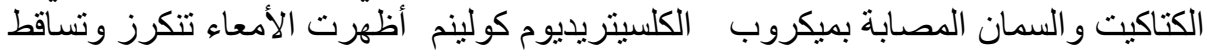

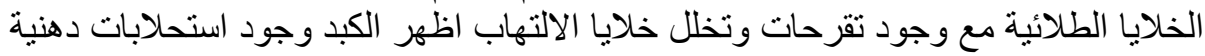

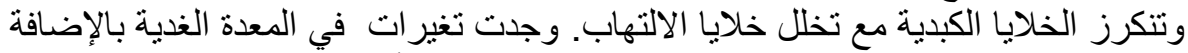

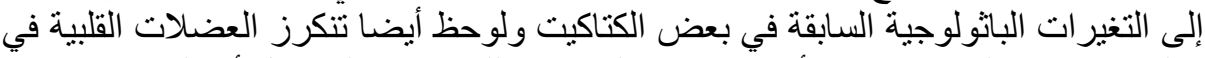

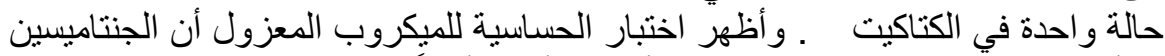
والأمبسلين وحمض النالاديكسيك هى الأدوية الأكثر تأثنيراً

\section{SUMMARY}

Forty samples (liver, spleen and intestine) from freshly dead quails and ninty five samples (liver, spleen and intestine) from freshly dead chikens (1-18 month age) were collected from different farms of Assiut governorate. Cl.colinum was isolated from quails and chickens at a rate of $70 \%$ and $27.4 \%$ respectively. Experimental infection of 5- week old quails intramuscular with isolated Cl.colinum from chickens revealed $100 \%$ mortality within 2-4 days postinoculation while $100 \%$ mortality 
within 8 days postinoculation was reported in orally infected quails. Intramuscular experimental infection of 12-day old chickens with isolated Cl.colinum caused $70 \%$ mortality within $48 \mathrm{~h}$. postinoculation. Reisolation of Cl.colinum from inoculated quails and chickens was successful. Histopathological study revealed that the intestine and liver were the most organs affected in chickens and quail infected. The intestine revealed epithelial necrosis desquamation, ulcerations and inflammatory cell infiltrations. The liver showed fatty degeneration, hepatocytic necrosis with moderate inflammatory cell infiltrations. Changes in glandular stomach were additionally reported in some infected chickens and myocardial necrosis was observed in one infected chicken. In vitro sensitivity test showed that gentamycin, ampicillin and naladixic acid were the most effective drugs.

Key words: Clostridium colinum, chickens, quails, experimental infection, histopathological study

\section{INTRODUCTION}

Ulcerative enteritis (U E) or quail disease, caused by Clostridium colinum (Cl. colinum) is a bacterial infection localized primarily in the in the intestinal tract. The disease occurs with epidemic proportions in quail, domestic fowl and in other avian hosts. and characterized by sudden onset and rapidly increasing mortality (Peckham 1960 and Berkhoff et al., 1974).

Quail disease was first reported in the united states in (1907) by Morse, several scattered outbreaks in quail were reported during the next 2 decades. Subsequently, infection in wild and domestic turkeys was discovered (Shillinger and Morley 1934 and Bullis et al., 1944). Other avian species found to be susceptible included pigeons (Clover 1951) and chickens (Graubmann and Grafner 1971). Bryant et al., (1973) cited that the $(\mathrm{U} E)$ is an important disease problem in some concentrated poultry - raising areas and is a threat to game birds either in Confinement or in the wild. Morries (1948) and peakham (1978) reported that C.colinum is considered the aetiological agent of (U E) in several avian species. Typically the affected chickens are between 4 and 15 weeks of age. Chronic carriers have been considered to be one of the most important factors in perpetuation of (U E). Berkhoff (1985) said that ulcerative enteritis is caused by a species of Clostridium named $\mathrm{Cl}$. Colinum on the basis of the $\mathrm{r}_{\mathrm{r}}$ RNA sequence analysis. Berkhoff and Campbell (1974). and Berkhoff et al. (1974) noticed that pure cultures of Cl.colinum grown anaerobically were highly pathogenic for quail 
following oral inoculation. The experimental disease appeared either in an acute form with birds dying around 3 days postinoculation (PI) or in a more chronic form with deaths occurring after 1-2 weeks. Under natural conditions, UE is transmitted through droppings, birds become infected by ingesting contaminated feed, water or litter. The organism produces spores resulting in permanent contamination of premises after an outbreak has occurred. $\mathrm{Cl}$. colinum the cause of quail disease, is referred as histotoxic Clostridia and includes the gas gangrene - producing Clostridia.

The organism is fastidious and primary isolation is difficult. Success has been reported in tryptose -phosphate- glucose broth with $8 \%$ sterile citrated horse plasma and thioglycollate broth with 3-10\% horse serum. In quail disease, Cl.colinum passes from the intestine via the portal circulation and lodges in the liver where diffuse liver necrosis is produced. The intestine becomes ulcerated and in some birds extensive necrosis of the spleen occurs. Affected birds are in active sluggish and anoxexic. They may die within 1-2 days due to replication of the Clostridia resulting in toxaemia, bacteraemia and often death. (Wages 2003).

The present study was taken to cover the following points:

- Isolation and identification of Cl.colinum from quails and chickens.

- Experimental infection of quails and broiler chickens with isolated Cl.colinum (which isolated from chickens).

- Histopathological study of naturally and experimentally infected quails and chickens.

- In vitro sensitivity test to show the most effective drugs against isolated organism.

\section{MATERIALS and METHODS}

\section{Materials:}

\section{Specimens:}

a - forty samples (liver, spleen and intestine) from freshly dead quails were collected from different parts of Assiut governorate.

b - Ninty five samples (liver, spleen and intestine) from freshly dead chickens (1-18 month age) collected from different farms of Assiut governorate.

\section{Media:}

Blood nutrient agar, tryptose phosphate agar and broth, semisolid agar, yeast extract, Esculin, ferric citrate, gelatin, glucose, mannose, sucrose, maltose, mannitol and lactose.

Reagents: 
Indole, phenol red and hydrogen peroxide.

Stain: Gram's stain.

\section{Pathogenicity test:}

a - Thiry, 5-week old quails were used.

b - Thirty, 12- day old balady chicks were used.

All birds were obtained from the faculty of Agriculuture farm Assiut University.

\section{In vitro antibiotic sensitivity discs used were:}

Oxytetracyclin $(30 \mu \mathrm{g})$, streptomycin $(10 \mu \mathrm{g})$, neomycin $(30 \mu \mathrm{g})$, penicillin $(10 \mu \mathrm{g})$, ampicillin $(10 \mu \mathrm{g})$, gentamycin $(10 \mu \mathrm{g})$, naladixic acid $(30 \mu \mathrm{g})$.

\section{Methods:}

\section{Isolation:}

Direct swab taken from liver, spleen and intestine of freshly dead birds (quails and chickens) and inoculated onto blood nutrient agar plates and onto tryptose - phosphate agar to which $0.2 \%$ glucose and $0.5 \%$ yeast extract were added, the $\mathrm{PH}$ is adjusted to 7.2 , then the medium is sterilized by autoclaving After cooling to $56^{\circ} \mathrm{c}, 8 \%$ horse plasma is added and the medium is poured into petridishes. Growth in broth media was prepared as stated previously but without agar and can be detected as early as 12-16 hours post inoculation (PI). All inoculated petridishes were incubated under anaerobic conditions in an anaerobic Jar for $24-48 \mathrm{~h}$. at $35-42{ }^{\circ} \mathrm{C}$. Suspected colonies were subjected to bacteriological examination to identify the organism by shawing (shape - size - colour) of the colonies, typical morphology of the organism by Gram's stain and studying the biochemical reactions.

\section{Pathogenicity test:}

\section{a - Quails:}

Thirty, 5- week old quails were used. Five from them were tested before the experiment and proved to be free from Cl.colinum. The other twenty five birds were divided as follow:

First group: Ten, 5- week old quails, they were inoculated intramuscularly with $0.2 \mathrm{ml}$ of a bacterial suspension of isolated Cl.colinum (from chickens) and containing $10^{7}$ $\mathrm{CFU} / \mathrm{ml}$ of $24-\mathrm{h}$ cultures in blood agar

Second group: Ten, 5 week old quails, they inoculated orally with $0.2 \mathrm{ml}$ of a bacterial suspension of Cl.colinum (which isolated from chickens) and containing $10^{7} \mathrm{CFU} / \mathrm{ml}$ of $24-\mathrm{h}$ cultures in blood agar. (The method was done according to Perelman et al., 1991). 
Third group: Five-5-week old quails, were left as control.

\section{b - Chicks:}

Thirty, 12-day-old balady chicks were used. Five from them were tested before the experiment and proved to be free from Cl.colinum. The other birds were divided as follow:

First group: Ten - 12-day-old, were infected intramuscular with $0.2 \mathrm{ml}$ of a bacterial suspension of Cl.colinum (which isolated from chicken) and containing $10^{5} \mathrm{CFU} / \mathrm{ml}$ of $24-\mathrm{h}$ culture in blood agar.

Second group: Ten, 12-day-old chicks, were inoculated orally with 0.2 $\mathrm{ml}$ of a bacterial suspension of Cl.colinum (which isolated from chickens) and containging $10^{5} \mathrm{CFU} / \mathrm{ml}$ of $24 \mathrm{~h}$. culture in blood agar. The method was done according to Perelman et al. (1991).

Third group: Five, 12 - day-old chicks were kept as control.

The three groups of chicken were kept in isolation units for a two - week period.

\section{In vitro antibiotic sensitivity test:}

Susceptibility test were carried out on blood nutrient agar using sensitivity discs.

\section{Histopathological examination:}

Small pieces of liver, spleen and intestine from naturally and experimentally infected quails and chickens were fixed in $10 \%$ buffered formalin, embedded in paraffin and stained with haematoxylin and eosin.

\section{RESULTS}

Postmortem examination (PM) of naturally infected quails revealed haemorrhagic enteritis in the duodenum and small punctate haemorrhages were visible through the serosa in the intestinal wall. There was mottling in the liver and congested enlarged spleen was found.

In naturally infected chickens PM examination showing also haemorrhagic enteritis and septicemia (congestion of all carcass and congestion and enlargement of liver, spleen and kidney).

Bacteriological examination in tryptose -phophate agar revealed that: the colonies were white circular, semitranslucent and had filamentous margins. 
On nutrient blood agar, the colonies were haemolytic. Growth in broth media showing actively growing cultures producing gas but gas continues for no more than 6-8 hours, after which growth settles to the bottom of the tube. Subculture was made from actively growing broth cultures still producing gas.

Gram's stain showing gram-positive rods with rounded ends, some have spores which are oval and subterminal. Sporogenic cells are much longer and thicker than non sporing cells.

Biochemical reactions revealed that the isolated organism was motile, esculin was hydrolyzed, fermented glucose, mannose, sucrose and maltose but not ferment lactose and mannitol. But was catalase, urease and indole negative and not liquefied gelatine.

According to cultural characters of the colonies, cellular morphology of the organism and biochemical reactions, the isolates proved to be Cl.colinum.

The frequency of the isolated Cl.colinum in quails was 28 out of 40 birds (70\%). In chickens was 26 out of 95 birds (27.4\%).

Pathogenicity test:

\section{a- Quails:}

Experimental infection of isolated organism to 5-weeks old quails intramuscular results in $100 \%$ mortality within $2-4$ days post inoculation (PI). PM examination characterized by congestion of the carcass (Fig 1) marked haemorrhagic enteritis in the duodenum (Fig.2), and small punctate haemorrhages were visible through the serosa in the intestinal wall (Fig.3). The intestine was filled with gases. (Fig.4). There was enlargement and mottling in the liver (Fig.5) and spleen. Inoculation of quails orally with isolated Cl.colinum caused $100 \%$ mortality within 8 days PI with signs and lesions similar to that described before.

There was no signs, lesions or death in control birds.

\section{b- chicks:}

Inoculation of 12- day old chicks by intramuscular injection caused $70 \%$ mortality within $48 \mathrm{~h}$. PI. Before death, the birds were listless with eyes partly closed with ruffling feathers. (Fig.6) PM examination revealed, the feed present in the crop and congestion of all carcass. (Fig.7). Two weeks after inoculation, the surviving birds. were sacrified, PM examination showed sever subcutaneous accumulation of serosanguinous fluid under the skin around the injection site (Fig8). Also there was accumulation of gases under the skin of breast (Fig.9) and in intestine. Precipitation of urates in the ureter were present in some birds with congestion of liver and kidney (Fig.10). Chicks which inoculated 
orally showing no death but revealed the same above lesions 2 weeks P.I but in mild form Reisolation of Cl.colinum from liver, spleen and intestine of inoculated quails and chicks was succeeded.

The effect of the different antibiotics on the isolated Cl.colinum are illustrated in Table 1.

\section{Histopathology:}

In chickens naturally infected with CL.colinum the intestine revealed extensive epithelial necrosis and desquamation. Inflammatory cell infiltration composed mostly of heterophils and mononuclear cells were observed in lamina propria (Fig.1). Focal aggregations of lymphocytes and macrophages were observed (Fig.2). The mucous glands also showed hypersecretory activity and necrobiotic changes (Fig.2). At some places mucosa had pealed off forming ulcers (Fig.3). The submucosa was oedematous and infiltrated with heterophils and mononuclear cells (Fig.4). The liver showed fatty degeneration and inflammatory cell infiltration composed of heterophils and mononuclear cells inbetween hepatic cords or in focal areas (Fig.5). Minute or large focal areas of hepatocytic necrosis was sometimes observed (Fig.5\&6). Many bacterial rods could be seen within macrophages and in hepatic sinusoids (Fig.7).

In experimentally infected chickens, the intestine and liver exhibited similar histopathological changes to those reported in natural cases in the present study. However, the glandular stomach in some cases revealed additional histopathological changes represented by necrobiotic changes in epithelial lining of mucosal glands (Fig.8). The lamina propria and submucosa infiltrated with heterophils and mononuclear cells (Fig. 8\&9). Many blood vessels in submucosa and serosa revealed endothelial degeneration (Fig.10).

In one case (experimentally infected) in addition to changes reported in the intestines and liver, the myocardium revealed coagulative necrosis (Fig.11).

In naturally infected quail, desquamation of the intestinal epithelium at many places was frequently observed (Fig.12). The intestinal mucosa at some places showed areas of necrosis with abundant nuclear debris and eosinophilic materials surrounded by mononuclear cells and heterophils (Fig.13). The liver revealed presence of necrotic foci with moderate inflammatory cell infiltration composed mainly of lymphocytes, macrophages and heterophils (Fig.14\&15). In experimentally infected quail, the intestinal epithelium was desquamated at some places. The intact enterocytes undergo coagulative necrosis (Fig.16). Focal and diffuse lymphocytic and heterophilic infiltration were observed in 
mucosa and sumucosa (Fig.16). Liver revealed coagulative necrosis of hepatocytes. Bacterial rods could be observed in hepatic sinusoid (Fig.17).

Table 1: Illustrate in vitro sensitivity test:

\begin{tabular}{|c|c|}
\hline Antibiotic discs & Sensitivity of C.colinum isolates \\
\hline Gentamycin & +++ \\
\hline Ampicillin & +++ \\
\hline Naladixic acid & +++ \\
\hline Penicillin & ++ \\
\hline Streptomycin & - \\
\hline Neomycin & - \\
\hline Oxytetracyclin & - \\
\hline+++ Highly sensitive & ++ Moderat sensitive \\
+ Weak sensitive & - Resistance
\end{tabular}


Assiut Vet. Med. J. Vol. 55 No. 122 July 2009

-9 - 
Assiut Vet. Med. J. Vol. 55 No. 122 July 2009

$-10-$ 
Assiut Vet. Med. J. Vol. 55 No. 122 July 2009

$-11-$ 


\section{LEGENDS FOR HISTOPATHOLOGICAL FIGURES}

Fig. 1: Intestine of a chick revealed extensive epithelial necrosis and desquamation. HE X100.

Fig. 2: Intestine of a chick shows hypersecretory activity and necrobiotic changes of mucous glands with focal aggregations of lymphocytes and macrophages. HE X400.

Fig. 3: Intestine reveals extensive epithelial necrosis and desquamation with ulcer formation. HE X400.

Fig. 4: Intestine of a chick reveals extensive epithelial necrosis and desquamation. The submucosa infiltrated with heterophils and mononuclear cells. HE X100.

Fig. 5: Liver of a chick showing fatty degeneration of hepatocytes. HE X100.

Fig. 6: Liver of a chick showing fatty degeneration of hepatocytes, presence of minute necrotic areas and inflammatory cell infiltration composed of heterophils and mononuclear cells inbetween hepatic cords. HE X400.

Fig. 7: Liver of a chick showing coagulastive necrosis of hepatocytes. Many bacterial rods could be seen within macrophages and in hepatic sinusoids.HE X400

Fig. 8: Glandular stomach of a chick showing necrobiotic changes in epithelial lining of mucosal glands and the lamina propria infiltrated with heterophils and mononuclear cells. HE X400.

Fig. 9: Glandular stomach of a chick showing that submucosa infiltrated with heterophils and mononuclear cells. HE X400.

Fig. 10: Glandular stomach of a chick showing that blood vessels in the submucosa revealed endothelial degeneration. HE X100.

Fig. 11: Myocardium of a chick revealed coagulative necrosis. HE X400.

Fig. 12: Intestine of a quail revealed desquamation of the intestinal epithelium at many places. HE X100.

Fig. 13: Intestine of a quail revealed area of necrosis with abundant nuclear debris and eosinophilic materials surrounded by mononuclear cells and heterophils. HE X100.

Fig. 14: liver of a quail revealed presence of necrotic foci. HE X100.

Fig. 15: Liver of a quail revealed moderate inflammatory cell infiltration composed mainly of lymphocytes, macrophages and heterophils. HE X400.

Fig. 16: Intestine of a quail showing desquamation of the intestinal epithelium. The intact enterocytes undergo coagulative necrosis. Focal and diffuse lymphocytic and heterophilic infiltration were observed in mucosa and sumucosa. HE X400.

Fig. 17: Liver of a quail showing coagulative necrosis of hepatocytes and presence of bacterial rods. HE X400. 
Assiut Vet. Med. J. Vol. 55 No. 122 July 2009

$-13-$ 
Assiut Vet. Med. J. Vol. 55 No. 122 July 2009

$-14-$ 
Assiut Vet. Med. J. Vol. 55 No. 122 July 2009

$-15-$ 
Assiut Vet. Med. J. Vol. 55 No. 122 July 2009

$-16-$ 


\section{DISCUSSION}

Ulcerative enteritis is a bacterial infection localized primarily in the intestinal tract. The disease occurs with epidemic proportions in quail, in domestic fowl and in other avian hosts. The diagnosis is based on gross lesions found in the intestine and liver. Ulcerative enteritis is most frequently encountered in chickens between 4 and 20 weeks of age with a mortality rate ranging from 1 to $10 \%$ (wages 2003).

In our study bacteriological examination of freshly dead quails revealed isolation of C.colinum from quails and chickens at a rate of $(70 \%)$ and $(27.4 \%)$ respectively. This rate in chickens is more less than that isolated by Perelman et al. (1991) who isolated Cl.colinum at rate of $(75 \%)$. 
Experimental infection of 5-week old quails intramuscular by isolated Cl.colinum (from chickens) reveald 100\% mortality within 2-4 days postinoculation, this is in agreement with the result of wages (2003) and Perelman et al. (1991). While in 12-day old chickens intramuscular injection caused $70 \%$ mortality within $48 \mathrm{~h}$. P1. This result is somewhat high than that observed by Perelman et al. (1991) who show $60 \%$ mortality. But our percentage is more high than that cited by wages (2003) which was ranged from 2-10\%.

Clinical signs of inoculated 5-week old quails intramuscular showing watery, white droppings, the birds were listless with eye partly closed and feathers dull and ruffled while gross lesions revealed marked haemorrhagic enteritis in the duodenum and small Punctate hemorrhages were visible through the serosa in the intestinal wall and the intestine was filled with gases, beside mottling and enlargement of liver and spleen, these results are in agreement with that observed by Perelman et al. (1991) Inoculation of quails orally with isolated C.colinum caused the same signs and lesions described before but after 8 days, P1.

Experimental infection of 12-day old chicks intramuscularly, the birds were listless with eye partly closed with ruffling feathers and the feed present in the crop. PM examination of dead birds showed congestion of all carcass and congestion and enlargement of liver, spleen and kidneys with hemorrhagic enteritis. The surviving birds showed sever subcutaneous accumulation of serosanguinous fluid under the skin around the infection site (turned to casiated material in some cases). Also there was accumulation of gases in the intestine and precipitation of urates in the ureter in some cases were present with congestion of liver and kidneys. Our result is in agreement with that reported by Perelman et al. (1991) and Wages (2003) But in our Experiment we show also accumulation of gases under the skin of breast. Chickens which inoculated orally showing no death and revealed the same above lesions 2 weeks P1 but in mild form, our result is differ with that cited by perelman et al. (1991) who say that chickens inoculated by oral route didn't develop any clinical signs or mortality.

We succeeded to reisolate Cl.colinum from experimentally infected birds and this result is in agreement with that observed by Perelman et al. (1991). The gross histopathological changes in the intestines and livers in the present study were somewhat similar to those observed in typical cases of ulcerative enteritis (Peckham, 1978, Perelman et al. (1991). In the present study, the absence of other diseases such as coccidiosis, Gumboro or hemorrhagic syndrome at least 
in experimentally infected birds (chickens and quail) suggested that infection of chickens or quail with C.colinum was a primary disease and not complication such as usually occurs in ulcerative enteritis (Davis, 1973, Peckham, 1978, Perelman). (1991)

The histopathological changes in the intestines and livers of experimentally infected chickens and quail in the present study were somewhat similar to those observed in natural corresponding cases in the same study. The later finding may intensify the later opinion that C.colinum causing ulcerative enteritis as a primary diseases in both species.

Based on the histopathological events in chickens and quail in the preent study it may conclude that C.colinum is important etiological agent causing serious diseases in both species.

In vitro sensitivity test we found that gentamycin, ampicillin and naladixic acid were the most effective drugs against isolated Cl.colinum but Perelman et al. (1991) show that ampicillin and penicillin were the most effective drugs.

Our conclusion in this study proved that the characterization of the isolates by their biochemical features and their extreme pathogenicity for quail confirms that the Clostridium isolated from chickens affected with syndrome belongs to the group of Cl.colinum. The fact that Cl.colinum was the only Clostridium consistently isolated strongly supports the idea that this bacterial is directly involved in the aetiology of this problem. Because the infectious organism is in the droppings and remains vialble indefinitely in letter (where survivous of an naturally infection may be carriers) it is recommended on problem farms to remove contaminated litter and use clean ones. In chickens avoid stresses caused by overcrowding, keep coccidiosis under control and use preventive measures against viral diseases which may act as stressors and / or cause immunosuppression.

\section{REFERENCES}

Perkhoff, H.A. (1985): Clostridium colinum sp. Nov. nom .rev., the causative agent of ulcerative enteritis (Quail disease) in quail chickens and pheasants. Int. J. syst Bacteriol. 35: 155-159.

Berkhoff, G.A and Campbell, S.G. (1974): Etiology and pathogenesis of ulcerative enteritis (Quail disease). The experimental disease. Avian Disease. 18, 205. 212. 
Berkhoff, G.A.; Campbell, S.G. and Nylor, H.B. (1974): Etiology and pathogenesis of ulcerative enteritis (Quail disease). Isolation of the causative anaerobe. Avian Dis. 18:186-194.

Berkhoff, G.A.; Campbell, S.G.; Naylor, H.B. and Smith, L.D. (1974): Etiology and pathogenesis of ulcerative enteritis (Quail disease). Characte. rization of the causative anaerob. Avian Dis. 18, 195- 204.

Bryant, E.S.; Gerencer, W.; Mallinson, E.T. and Stein, G. (1973): Report of the committee on nomenclature and reporting of disease. Northeastern Conference on Avian Dis. 17: 904-911.

Bullis, K.L. and Van Roekel, H. (1944): Uncommon pathological conditions in chickens and turkeys. Cornell Vet. 34: 312 -319.

Glover, J.S. (1951): Ulcerative enteritis in pigeons. Can. J. comp Med Vet. Sci. 15: 295-297.

Graub Mann H.D. and Grafner, G. (1971): Ulcerative enteritis in the chicken (Quail disease) occurrence and pathology. Monatsh veterinarmed. 26 (23): 903-907.

Morries, J.A. (1948): The use of the complement fixation test in the detection of ulcerative enteritis in quail. Am. J. Vet. Sc. 9: 102-103.

Morse, G.B. (1907): Quail disease in the united stats united states Department of Agriculture BAI Circ 109.

Peckham, M.C. (1960): Further studies on the causative organism of ulcerative enteritis. Avian Dis. 4:449-456.

Perelman, B.; Mints, S.; Zjut, M.; Kuttin, E. and Machny, S. (1991): An unusual Clostridium colinum infection in broiler chickens. Avian path. 20, 475-480.

Shillinger, J.E. and Morley, L.C. (1943): Studies on ulcerative enteritis in quail. J. Am. Vet. Med. Assoc. 84: 25-35.

Wages, D.P. (2003): Ulcerative enteritis (Quail disease) In: Diseases of Poultry edited by: Calnek, B.W; Barnes, H. J.; Mcdouglad, C. W. and Saif, Y.M.P. 776-780. 
Fig. 1: Showing congestion of the carcass of experimentally infecte quail.

Fig. 2: Showing haemorrhagic enteritis in experimentally infected quail.

Fig. 3: Small punctate haemorrhages are visible through the serosa in the intestinal wall of experimentally infected quail
Fig. 4: The intestine was filled with gases.

Fig. 5: Enlargement and mottling in the liver of experimentally infected quail.

Fig. 6: Experimentally infected chick is listless with eyes partly closed with rufflung feathers.

Fig. 7: Experimentally infected chick Fig. 8: Experimentally infected showing the feed present in the crop with congestion of all carcass. chick showing caseated material under the sking around the injection site.

Fig. 9: Showing accumulation of gases under the skin of breast in experimentally infected chick.

Fig. 10: Showing congestion and mottling of liver of experimentally infected chick. 
Assiut Vet. Med. J. Vol. 55 No. 122 July 2009

$-22-$ 